This item was submitted to Loughborough's Research Repository by the author.

Items in Figshare are protected by copyright, with all rights reserved, unless otherwise indicated.

\title{
Simulation of particle flow in an inertial particle separator with an Eulerian velocity re-associated two-node quadrature-based method of moments
}

PLEASE CITE THE PUBLISHED VERSION

http://dx.doi.org/10.2514/6.2016-1641

\section{PUBLISHER}

AIAA / @ The Authors

VERSION

AM (Accepted Manuscript)

\section{PUBLISHER STATEMENT}

This work is made available according to the conditions of the Creative Commons Attribution-NonCommercialNoDerivatives 4.0 International (CC BY-NC-ND 4.0) licence. Full details of this licence are available at: https://creativecommons.org/licenses/by-nc-nd/4.0/

\section{LICENCE}

CC BY-NC-ND 4.0

\section{REPOSITORY RECORD}

Sun, Dan, Andrew Garmory, Gary J. Page, and Indi H. Tristanto. 2016. "Simulation of Particle Flow in an Inertial Particle Separator with an Eulerian Velocity Re-associated Two-node Quadrature-based Method of Moments". figshare. https://hdl.handle.net/2134/22499. 


\title{
Simulation of Particle flow in an Inertial Particle Separator with an Eulerian Velocity Re-Associated Two-Node Quadrature-Based Method of Moments
}

\author{
Dan Sun $^{1}$, Andrew Garmory ${ }^{2}$ and Gary J. Page ${ }^{3}$ \\ Department of Aeronautical and Automotive Engineering, Loughborough, LE11 3TU, UK \\ Indi Tristanto 4 \\ Rolls-Royce PLC. Derby, DE24 8BJ, UK
}

This paper presents research into practical simulations of particle flow in inertial particle separators (IPS) for helicopters and tilt rotor aircraft. The flow field of the carrier gas is predicted by means of the two-equation $k-\varepsilon$ turbulence model. An Eulerian methodology is used to trace the particle trajectories of foreign particles such as droplets, ice and sand. To predict the characteristics of particle wall bouncing in dilute particle flow, the velocity re-associated two-node quadrature-based method of moments (VR-QMOM) is used. The particle distribution in the IPS is predicted for various particle sizes and these are compared with results from a Lagrangian particle tracking method. The particle-wall interactions and the separation efficiencies are studied for solid particles bouncing off perfectly elastic walls and an IPS shell coated with the M246 alloy which changes the coefficients of restitution. The simulated separation efficiencies predicted by the Eulerian method are compared with the simulation using the Lagrangian method over a range of particle sizes. The VR-QMOM method is seen to reproduce the particle bouncing and trajectory crossing behavior and to agree well with the Lagrangian method for predicted separation efficiencies. The new VR-QMOM method is shown to be an accurate and convenient alternative to established Lagrangian approaches.

\section{Nomenclature}

$a_{i j} \quad=$ second-order moment of velocity deviation

$a_{i i i} \quad=$ third-order moment of velocity deviation

$C=$ particle concentration

$C_{D} \quad=$ particle drag coefficient

C $=$ collision term

$d_{p} \quad=$ diameter of particles

$\mathrm{e}_{\mathrm{w}, \mathrm{t}} \quad=$ restitution coefficient of wall particle interaction at tangential direction

$\mathrm{e}_{\mathrm{w}, \mathrm{n}} \quad=$ restitution coefficient of wall particle interaction at normal direction

$\mathbf{F} \quad=$ external force vector

$f=$ velocity distribution function

$m_{p} \quad=$ mass of single particle

$M^{s}{ }_{i j . .} \quad=$ s-order moment of velocities

$n \quad=$ number density of particles

$\operatorname{Re}_{p} \quad=$ particle Reynolds number

$\mathbf{s}_{\mathbf{p}} \quad=$ solid phase stress

$\mathbf{U}_{\mathbf{f}} \quad=$ velocity vector of fluid phase

$\mathbf{U}=$ Velocity vector of particles

$\mathbf{U}_{1} \quad=\quad$ velocity vector of node 1 particles

$\mathbf{U}_{2} \quad=$ velocity vector of node 2 particles

1 Corresponding Author: Research Associate, AIAA Member, email: d.sun@lboro.ac.uk

2 Lecturer, AIAA Member

3 Senior Lecturer, AIAA Senior Member

4 Currently a Royal Society Industry Fellow at Loughborough University, AIAA Senior Member

1

American Institute of Aeronautics and Astronautics 


$\begin{array}{ll}\mathbf{U}_{\mathbf{p}} & =\text { mean particle velocity vector of node } 1 \text { and node } 2 \\ \rho_{p} & =\text { density of particles } \\ \rho_{f} & =\text { fluid density } \\ \tau_{\mathrm{p}} & =\text { characteristic time for relaxation to fluid velocity } \\ \mu_{f} & =\text { kinematic viscosity of fluid phase } \\ \eta & =\text { separation efficiency }\end{array}$

\section{Introduction}

Helicopters and turbo-prop driven transport aircraft often operate in harsh environments. During take-off and landing, the downwash of the rotor can lift particles off the ground where they may be ingested by the engine. In these brownout conditions, the pilot cannot see the nearby object and an accident may be caused ${ }^{1}$. Furthermore, the engine internal components are at a high risk of being exposed to the ingested particles that leads to a reduction of the engine lifetime and an increase in fuel consumption ${ }^{2}$. Sand ingestion potentially causes erosion of the compressor blades, contamination of the engine's oil system and blockage of the cooling systems. This leads to a loss of power and a reduction in surge margins. In the 1960's, the average operation time of helicopters in critical environments was only 300 hours due to erosion, and some helicopter engines

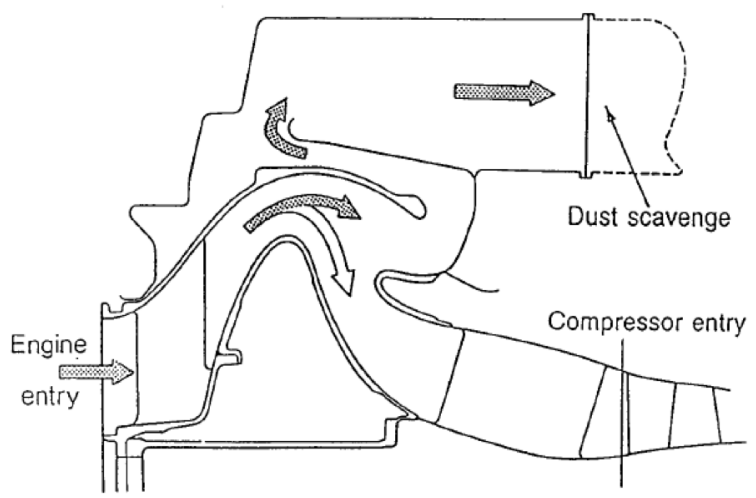

Figure 1. Inertial particle separator ${ }^{9}$ operated in jungle environments had to be removed in $100 \mathrm{hrs}^{3}$. Also in desert environments, the engines were removed after fewer than 100 hours and even 20 hours flying ${ }^{4}$. Although sand ingestion is mostly prevalent when helicopter and tilt rotor aircraft operate in ground effect, particles such as volcanic ash or ice crystal may be present at high altitude which poses a threat to the safe operation of commercial aircraft ${ }^{5,6}$. The erosion from the volcanic ash is four times worse than the damage from the quartz sand typically found on the desert surface ${ }^{7}$. Furthermore, this puts a requirement to be able to trace the particles accurately over a long distance into the engine core through 10-20 compressor stages. The present paper is focused on the more classical problem of sand ingestion in ground effect operation of helicopter and tilt rotor aircraft. Although this class of problem usually refers to relatively coarse grain of sand, the current study also investigates ingestion of ash particles that have finer grain, as a proof of concept for modelling volcanic ash ingestion into the engine.

Although erosion-resistant coatings for the blades are able to prolong the operational life of a helicopter engine in the desert $^{8}$, including an inertial particle separator (IPS) as part of the engine intake remain necessary as it that has proven to be a successful method of protecting the engine core. These have relatively low pressure loss and low weight, making them suitable for helicopters, while preventing the foreign particles entering the gas turbine. The main geometry for the IPS design consists of the hub surface, outer surface, shroud and splitter nose. Using the inertial characteristics of particles passing through the complex geometry, the particles will leave the core and enter the scavenge flow path, and thus the foreign particles are separated from the gas flow path, as shown in Fig. 1 that was taken from ref. 9.

An IPS relies on the particle inertia in order to clean the airflow into the engine core. Since the IPS has a restricted volume the inertia dominated particles flight would lead to trajectory multiple rebound with the IPS geometry before exiting the domain. Thus, wall interaction model is an integral part of designing the IPS geometry in order to manipulate the particle trajectory towards the scavenge duct. In soft surface interaction, either due to the nature of the particles (e.g. water droplets) or liquid film covered surfaces, the energy loss through deformation may lead the particle to stick or have a much weaker rebound. Interaction between sand particle and IPS surfaces, which are generally dry, is having hard surface characteristic with little deformation and therefore the rebound and incoming momenta have similar magnitude. Even if the particle shattered during the wall interaction, the smaller pieces are often flying off the wall with high momentum.

The ground sand found in brownout conditions are mostly silica $\left(\mathrm{SiO}_{2}\right)$, with a large range of particle size distribution from microns to millimeters ${ }^{10}$. In Fig. 2, the weighted distributions of particle size are shown. The distributions of C-Spec $(40-800 \mu \mathrm{m})^{11}$ and AC-Coarse $(1-200 \mu \mathrm{m})^{12}$ are typically used for studies of foreign particle 
ingestion in desert operation. The volcanic ash comprises various types of materials including rock and metal and the particle size will vary with distance from the volcanic eruption. At high altitude, the ash cloud mainly consists of fine particles, which can easily deposit on the surface of the internal components of the engine in large numbers. The blue PSD in Fig. 2 shows the fine ash distribution $(1-25 \mu \mathrm{m}){ }^{13}$ from the coal burning process which can be used a guide to that may be expected in volcanic ash.

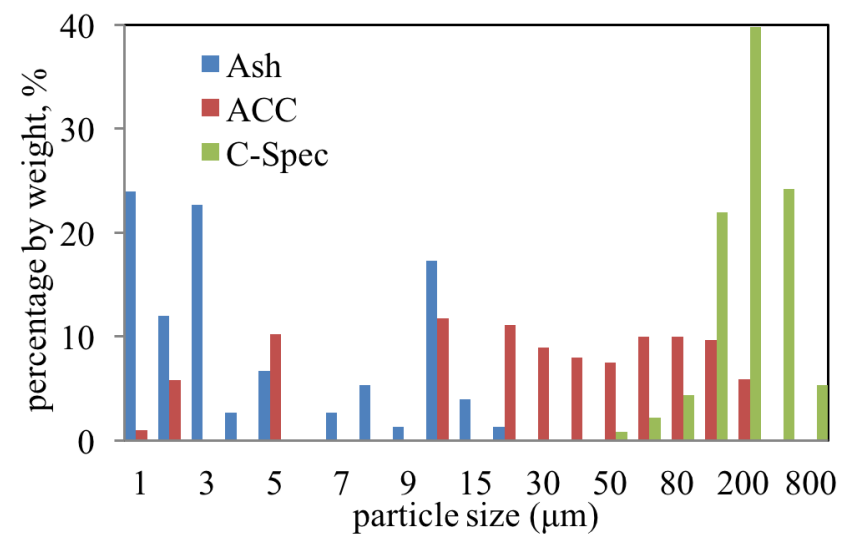

\section{Figure 2. Particle size spectrum. ( C_Spec, 40-800 $\mu \mathrm{m}^{11}$, AC Coarse, 1-200 $\mu \mathrm{m}^{12}$, and coal-burning ash, $1-25 \mu \mathbf{m}^{13}$ ).}

The fundamental dynamics of the multiphase flow in IPS have been investigated over decades by means of experiments and computational methods ${ }^{14,15}$. The particle dynamics for a specific design of IPS can be studied by experiment and the particle bounce characteristics can be investigated by testing the particle trajectories before and after collision with the wall, which then used to generate an empirical wall bouncing model ${ }^{16,17,18}$. CFD can be a flexible and efficient tool to achieve the optimal configuration of components. The Lagrangian method with empirical wall bouncing model is usually used within a CFD calculation to predict particle trajectories and evaluate the separation efficiency of the inertial particle separators. Calzada et al ${ }^{19}$ predicted the particle trajectories of dust at diameters from $1 \mu \mathrm{m}$ to $500 \mu \mathrm{m}$. For the conditions they investigated particles with diameter larger than $20 \mu \mathrm{m}$ could be totally separated by the IPS tested. The $10 \mu \mathrm{m}$ particles partly entered the core flow path. The $1 \mu \mathrm{m}$ particles followed the gas flow path and were hard to be separated.

The Lagrangian method has long been established as an accurate way of modelling dilute particle with relatively low computational cost. However, in practice, the total simulation cost may be high if a large number of particles must be tracked in order to build a statistically meaningful sample of particle deposition in the zone of interest. This is particularly true when the simulation is aimed at ingestion deep within the gas turbine core, for instance volcanic ash in the HP compression system, since the parallelization schemes of the Lagrangian method are not as efficient as those for Eulerian methods. Lagrangian methods are also difficult to use efficiently with multiple rotating and stationary frames of reference as found in turbomachinery. Furthermore, Lagrangian based methods may require manual interaction by the user to position particle injectors in order to ensure enough particles are deposited in the zone of interest that lies after a series of rotor-stator blade rows. Hence it is not inconceivable that the total cost of performing Lagrangian simulation can increase by more than 3 order of magnitude when going form a single turbomachine zone with simple topology - representing an inlet or a single blade row - to a complete turbomachine stage, whereas the Eulerian based equivalent would increase more linearly with the number of blade rows, i.e. around 1 order of magnitude. For these reasons an Eulerian based multiphase method offers the potential to improve simulations of particle laden flow in aircraft engines. Traditional Eulerian methods are not able to calculate particle bouncing if only phase mean velocity is available within a cell. However, the Eulerian method of velocity-based quadrature-based method of moments $(\mathrm{QMOM})^{20}$ shows the capability to simulate particle trajectory crossing and wall bouncing flow at dilute particle concentration ${ }^{21,22}$. A new robust QMOM method, velocity re-associated quadrature-based method of moment (VR-QMOM) has been developed in recent research which is able to predict the arbitrary crossing trajectories and wall-bouncing of a dilute particle flow ${ }^{23}$. In VR-QMOM, the two-node quadrature approach used gives high computational efficiency compared to QMOM models using more nodes ${ }^{20,21}$, and the new thirteen-moment system overcomes the problem of failed prediction at some specific conditions when using the two-node eight-moment QMOM model by Desjardins ${ }^{22}$.

The aim of this research is to establish an Eulerian-Eulerian two-phase method that is capable of predicting the multiphase flow in the IPS. This is part of a wider project whose intention is to develop methods to trace the particle 
flow through the core of the engine using a computationally efficient Eulerian method. In this paper the Eulerian method VR-QMOM is used to investigate the particle separation behavior at various particle sizes that may damage the gas turbine performances. Results are compared to those computed using a Lagrangian technique in order to demonstrate the viability of the VR-QMOM model when predicting flows with wall bouncing. Since an IPS has relatively simple topology, the cost of modelling is mainly related to the computing time, in which case the Lagrangian model is computationally cheaper than any Eulerian based method.

\section{Methodologies}

The dynamics of the particle flow in the inertial particle separator are determined by the inertial characteristics of the particles and their interaction with the fluid field. The simulations in this work are carried out in the EulerianLagrangian and Eulerian-Eulerian multiphase framework. The gas flow field is predicted by the Reynolds Averaged Navier-Stokes equations together with a two-equation $\mathrm{k}-\varepsilon$ turbulence model with standard wall functions ${ }^{24}$. The particle flow under the dilute flow regime is modeled by an Eulerian method (VR-QMOM) and, for comparison, a Lagrangian method. The multiphase simulations with the Lagrangian method for particle flow are carried out with the commercial software ANSYS Fluent. The velocity re-associated two-node quadrature method of moment (VRQMOM $)^{23}$ is implemented into an unstructured, industrial CFD tool developed particularly for turbomachinery, the Rolls-Royce Hydra CFD code ${ }^{25}$.

The present simulation is of dilute particle concentration, where the inter-particle collisions can be neglected and one-way coupling is assumed for the interaction between the gas and particle phase. It also assumed that the particles in the simulation are uniform and spherical. The basic Eulerian method and the new VR-QMOM model for particles are briefly presented here.

\section{A. Basic Eulerian method}

The basic Eulerian method cannot predict bouncing flow at dilute particle concentration. Taking the dispersed particle phase as a continuum, the particle concentration $C$ is the variable used to represent the percentage of the particles dispersed locally in the fluid phase. The flux transportation of the particle phase is expressed by the mean velocity of particles. The transport equations for the particle phase are presented briefly by the continuity equation and momentum equation, seen as follows.

$$
\begin{gathered}
\frac{\partial\left(\rho_{p} C\right)}{\partial t}+\nabla \cdot\left(\rho_{p} C \mathbf{U}_{\boldsymbol{p}}\right)=0 \\
\frac{\partial\left(\rho_{p} C \mathbf{U}_{p}\right)}{\partial t}+\nabla \cdot\left(\rho_{p} C \mathbf{U}_{\boldsymbol{p}} \mathbf{U}_{\boldsymbol{p}}\right)=\nabla \cdot \mathbf{s}_{\boldsymbol{p}}+\mathbf{F}
\end{gathered}
$$

where $\mathbf{U}_{\mathbf{p}}$ is the mean velocity vector of the dispersed particle phase. $\mathbf{s}_{\mathbf{p}}$ is the solid phase stress, which can be neglected in dilute flow, $\mathbf{F}$ is the external force vector, may including the effect of gravity, drag forces, electrostatic forces, lift force, thermophoresis and turbophoresis ${ }^{26}$.

The Neumann boundary condition can be used at the wall for the simulation of solid particles. This boundary treatment represents particle deposition on the wall, which is largely representative for 'softer' particle such as liquid droplets. This boundary is not easily extensible to hard particle simulation where wall bouncing is a key phenomenon, even when an impact source term is added to the momentum equation in order to model the change of particle momentum at the wall.

\section{B. VR-QMOM for solid particles}

The new VR-QMOM model used here is a two-node approach based on the velocity-distributed quadrature moment method ${ }^{23}$. The transport equations are first established by the kinetic theory of gases

$$
\frac{\partial f}{\partial t}+\mathbf{U} \cdot \frac{\partial f}{\partial \mathbf{x}}+\frac{\partial f}{\partial U}\left(f \frac{\mathbf{F}}{m_{p}}\right)=\mathbb{C}
$$

where $f$ is the velocity distribution function which give the possibility(density) of the particles near a specific velocity. $\mathbf{U}$ is the particle velocity vector. $\mathbb{C}$ is the inter-particle collision term, where $\mathbb{C}=0$ for dilute particle flow and hence is ignored in this work. $\mathbf{F}$ in the last term on the LHS is external force vector, considering the drag force in this study, 


$$
\mathbf{F}\left(\mathbf{U}_{\mathbf{f}}, \mathbf{U}\right)=\frac{m_{p}}{\tau_{p}}\left(\mathbf{U}_{\mathbf{f}}-\mathbf{U}\right)
$$

where $\tau_{p}$ is the characteristic time for relaxation of particle velocity to fluid velocity due to aerodynamic drag,

$$
\frac{1}{\tau_{p}}=\frac{3 \rho_{f}}{4 d_{p} \rho_{p}} C_{D}\left|\mathbf{U}_{\mathbf{f}}-\mathbf{U}\right|
$$

where $\rho_{f}$ is the fluid density, $\rho_{p}$ is the particle density, $d_{p}$ is the particle diameter, and $\mathbf{U}_{\mathbf{f}}$ is the fluid velocity vector. $C_{D}$ is the particle drag coefficient, which in this work is given by the Schiller-Nauman correlation for small spheres when $1 \leq R e_{P} \leq 1000^{27}$,

$$
C_{D}=\frac{24}{R e_{p}}\left(1+0.15 R e_{P}^{0.687}\right)
$$

and $R e_{P}=\rho_{f} d_{p}\left|\mathbf{U}_{\mathbf{f}}-\mathbf{U}\right| / \mu_{f}$ is particle Reynolds number, where $\mu_{f}$ is the kinematic viscosity of the fluid phase.

In the two-node quadrature approach, the distribution of the velocity fields can be denoted by the number density $n$ and velocity $\mathbf{U}$, which are the weights $\left(n_{1}, n_{2}\right)$ and $\operatorname{abscissas}\left(\mathbf{U}_{1}, \mathbf{U}_{2}\right)$ of the two nodes. Using delta-functions to represent the dispersed distribution of particle velocity,

$$
f=n_{1} \delta\left(\mathbf{U}-\mathbf{U}_{\mathbf{1}}\right)+n_{2} \delta\left(\mathbf{U}-\mathbf{U}_{2}\right)
$$

The arbitrary moment of velocity can be expressed as,

$$
M_{i j \ldots l}^{S}=n_{1} U_{1 i} U_{1 j} \cdots U_{1 l}+n_{2} U_{2 i} U_{2 j} \cdots U_{2 l}
$$

Then the moment transport equations for particles can be obtained from Eq (3) as,

$$
\begin{gathered}
\frac{\partial M^{0}}{\partial t}+\frac{\partial M_{k}^{1}}{\partial x_{k}}=0 \\
\frac{\partial M_{i}^{1}}{\partial t}+\frac{\partial M_{i k}^{2}}{\partial x_{k}}=\frac{n_{1}}{m_{p}} F_{1, i}+\frac{n_{2}}{m_{p}} F_{2, i} \\
\frac{\partial M_{i j}^{2}}{\partial t}+\frac{\partial M_{i j k}^{3}}{\partial x_{k}}=\frac{n_{1}}{m_{p}} U_{1, i} F_{1, j}+\frac{n_{1}}{m_{p}} U_{1, j} F_{1, i}+\frac{n_{2}}{m_{p}} U_{2, i} F_{2, j}+\frac{n_{2}}{m_{p}} U_{2, j} F_{2, i} \\
\frac{\partial M_{i i i}^{3}}{\partial t}+\frac{\partial M_{i i k k}^{4}}{\partial x_{k}}=3 \frac{n_{1}}{m_{p}}\left(U_{1, i}\right)^{2} F_{1, i}+3 \frac{n_{2}}{m_{p}}\left(U_{2, i}\right)^{2} F_{2, i}
\end{gathered}
$$

The terms on the right hand side represent interactions due to the influence from the interactions with fluid phase. In VR-QMOM, the moment equations (9) are solved for 13 moments in a three dimensional system, these are

$$
W^{3}=\left(M^{0}, M_{i}^{1}, M_{j}^{1}, M_{k}^{1}, M_{i i}^{2}, M_{j j}^{2}, M_{k k}^{2}, M_{i j}^{2}, M_{j k}^{2}, M_{i k}^{2}, M_{i i i}^{3}, M_{j j j}^{3}, M_{k k k}^{3}\right) \in \mathbb{R}^{13} .
$$

Based on the two-node definition of the moments given in Equation (7) the transport equation for an arbitrary one of these 13 moments $M_{a b}$ can be written in terms of the velocity fields of the two nodes

$$
\frac{\partial M_{a b}}{\partial t}+\frac{\partial n_{1} U_{1 a} U_{1 b} U_{1 i}}{\partial x_{i}}+\frac{\partial n_{2} U_{2 a} U_{2 b} U_{2 i}}{\partial x_{i}}=S
$$

where $S$ is the source term such as drag. The key to the QMOM approach is that once the moments have been updated it is necessary to recover the updated nodal velocity fields and their weightings. In the closure approach of VR-QMOM, the first step is to calculate the abscissas and weights with the product-difference (PD) algorithm, as in the established third-order QMOM model ${ }^{20}$. The mean velocity of particles in each direction is found by $U_{p i}=$ $M_{i}^{1} / M^{0}$. This can be used to define the covariance matrix and the third order velocity variance vector 


$$
\begin{gathered}
{\left[a_{i j}\right]=\left[\begin{array}{ccc}
M_{i i}^{2} / M^{0}-U_{p i} U_{p i} & M_{i j}^{2} / M^{0}-U_{p i} U_{p j} & M_{i k}^{2} / M^{0}-U_{p i} U_{p k} \\
M_{i j}^{2} / M^{0}-U_{p i} U_{p j} & M_{j j}^{2} / M^{0}-U_{p j} U_{p j} & M_{j k}^{2} / M^{0}-U_{p j} U_{p k} \\
M_{i k}^{2} / M^{0}-U_{p i} U_{p k} & M_{j k}^{2} / M^{0}-U_{p j} U_{p k} & M_{k k}^{2} / M^{0}-U_{p k} U_{p k}
\end{array}\right]} \\
{\left[a_{i i i}\right]=\left[\begin{array}{l}
\frac{1}{M_{0}} M_{i i i}-U_{p i}{ }^{3}-3 a_{p i}{ }^{2} U_{p i} \\
\frac{1}{M_{0}} M_{j j j}-U_{p j}{ }^{3}-3 a_{p j}{ }^{2} U_{p j} \\
\frac{1}{M_{0}} M_{k k k}-U_{p k}{ }^{3}-3 a_{p k}{ }^{2} U_{p k}
\end{array}\right]}
\end{gathered}
$$

The node weights can be calculated using

$$
\begin{aligned}
& x_{i}=\frac{a_{i i i}}{2 \sqrt{a_{i i i}{ }^{2}+4 a_{i i}{ }^{3}}} \\
& n_{1,2}=\left(\frac{1}{2} \pm x_{i}\right) M_{0}
\end{aligned}
$$

The velocity components in the $i$-direction for the two nodes are then found from

$$
\begin{aligned}
& U_{1 i}=U_{p i}-\left(\sqrt{a_{i i}}\right) \sqrt{\frac{1 / 2-x_{i}}{1 / 2+x_{i}}} \\
& U_{2 i}=U_{p i}+\left(\sqrt{a_{i i}}\right) \sqrt{\frac{1 / 2+x_{i}}{1 / 2-x_{i}}} .
\end{aligned}
$$

The components in the $j$ and $k$ directions can be found by the same procedure. However, in order to ensure correct physical behavior for all trajectory crossing cases it is necessary to ensure that the velocity components in each node are correctly associated with each other. Therefore, the second step is to set up the correlation between these abscissas and weights in order to set the final two velocity components for each flow field. The key issue of the model is to re-associate the relationship between $\left(U_{-j}, U_{+j}, U_{-k}, U_{+k}\right)$ and $\left(n_{1}, \mathrm{n}_{2}, U_{1 i}, U_{2 i}\right)$ using the second order cross moments of the velocity of particles. Therefore, the value of the remaining four velocities can be recalculated by the second order cross moment of velocity derivation.

$$
\begin{aligned}
& U_{1 j}=U_{p j}-\left(\frac{a_{i j}}{\sqrt{a_{i i}}}\right) \sqrt{\frac{n_{2}}{n_{1}}} \\
& U_{2 j}=U_{p j}+\left(\frac{a_{i j}}{\sqrt{a_{i i}}}\right) \sqrt{\frac{n_{1}}{n_{2}}} \\
& U_{1 k}=U_{p k}-\left(\frac{a_{i k}}{\sqrt{a_{i i}}}\right) \sqrt{\frac{n_{2}}{n_{1}}} \\
& U_{2 k}=U_{p k}+\left(\frac{a_{i k}}{\sqrt{a_{i i}}}\right) \sqrt{\frac{n_{1}}{n_{2}}}
\end{aligned}
$$

A unique pair of weighted velocity fields is thus obtained from the set of moments. Thus, the velocities of the two nodes are re-associated by the cross-moments of second-order. While the method as described above does not use all 13 moments, in practice we solve for them all to ensure redundancy for the case of $a_{i i}=0$.

To give the desired surface bouncing behavior it is necessary to specify appropriate boundary conditions for the dispersed phase. In this research, we assume that the particle bouncing can be described by deterministic coefficients of restitution in the normal and tangential directions. This can be applied for both nodal velocity fields.

$$
\left[\begin{array}{c}
n_{\alpha} \\
U_{\alpha n} \\
U_{\alpha t}
\end{array}\right]_{b c}=\left[\begin{array}{c}
n_{\alpha} / e_{t} \\
-e_{w n} U_{\alpha n} \\
e_{w t} U_{\alpha t}
\end{array}\right]_{f}
$$

Where the subscript $n$ is for normal direction of the wall and $t$ is for tangential direction. $e_{t}$ is the total velocity coefficient of restitution defined as the ratio of velocity of reflected particles to injecting particles. This method allows the present model to utilize the existing empirical wall bouncing model that had been devised primarily for Lagrangian based particle simulation. 
The VR-QMOM model is implemented in to the Rolls-Royce Hydra CFD code ${ }^{25}$ which uses an unstructured mesh formulation with median dual control volumes. The transport step of the QMOM requires the moments to be updated using the weighted velocity nodes. A Finite Volume method is used to construct the fluxes in the unstructured discretization scheme. Currently, for robustness, a first-order upwind scheme is used in the simulation with VR-QMOM $M_{\alpha, f}=M_{\alpha, U P}$.

\section{Simulation and Results}

\section{A. Particle wall bouncing prediction}

Figure 3 shows the particle concentration predicted by the existing basic Eulerian method introduced in section II-A. When the particles hit the surface of the IPS, they will lose all the momentum in the normal direction. In the inlet region, all the particles at the wall flow along the surface, and leave the wall at the positive curvature driven by the inertial characteristic of the particles, i.e. the particles near the hub surface at the shroud and the particles entering the scavenge path.

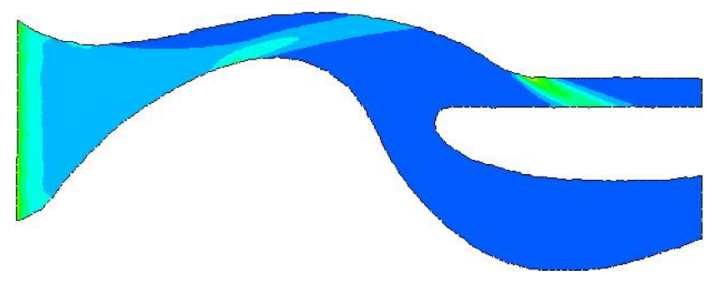

Figure 3. Particle concentration of $200 \mu \mathrm{m}$ sand in IPS by basic Eulerian method

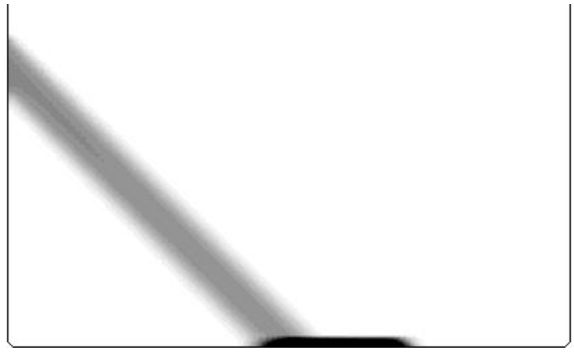

a) existing basic Eulerian method

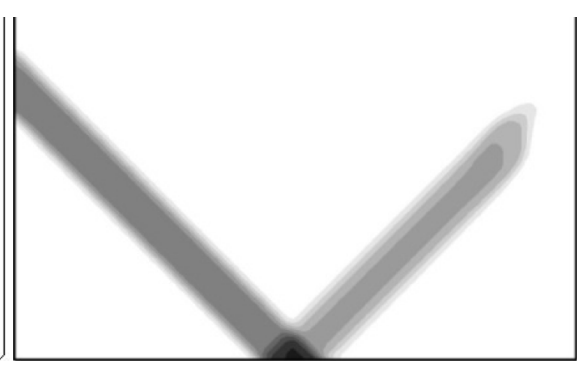

(b) new VR-QMOM

Figure 4. Particle concentration of wall bouncing flow

In Fig. 4(a), the particle concentration is simulated by the traditional Eulerian method. The single velocity field averages the particle velocities of injecting particles and reflected particles, making the mean velocity near to zero normal to the wall, such that the particles will flow along the surface. Figure 4(b) shows a simulation using the new VR-QMOM method. As two velocity fields or nodes are used to represent the incoming and outgoing particles at the surface, the bouncing flow is able to be predicted successfully. The arbitrary crossing-trajectories of the two particle streams and multiphase simulation have been studied and validated in previous research ${ }^{23}$.

The particle-surface interaction mechanism is defined with the boundary conditions and the restitution coefficients as described in Eq (19). In VR-QMOM, the particle velocities at the surface are described by the two velocity fields. Therefore, the rebounded particle velocities are calculated directly from the injection particles. Figure 5 shows the prediction of the particle wall bouncing flow with varying restitution coefficients. The particle beams can be seen to exactly follow the expected analytical rebound angles as shown by the dotted lines. 


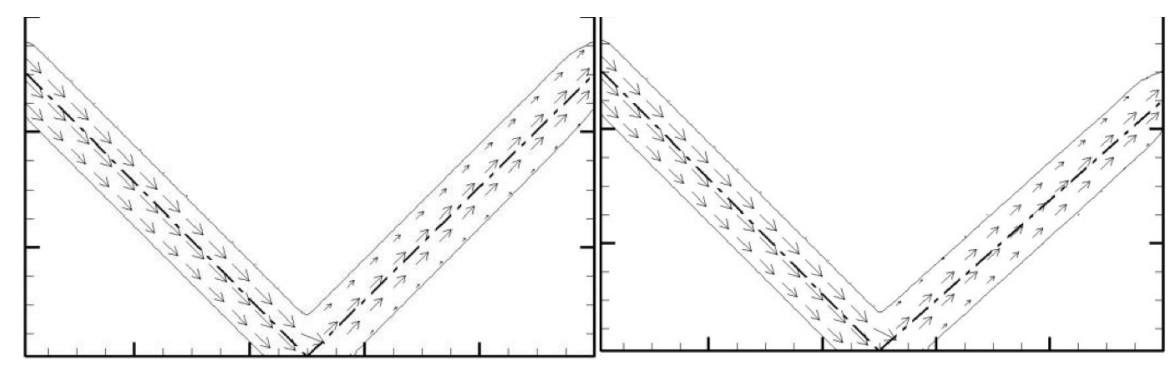

a) $e_{w t}=1.0$

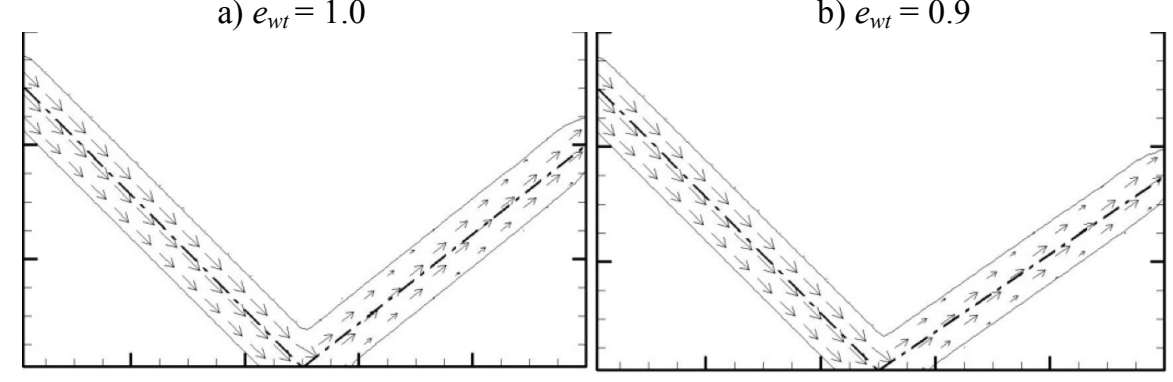

c) $e_{w t}=0.8$

d) $e_{w t}=0.7$

Figure 5. Particle paths predicted by VR-QMOM at various tangential restitution coefficients. Expected rebound path shown as dotted line.

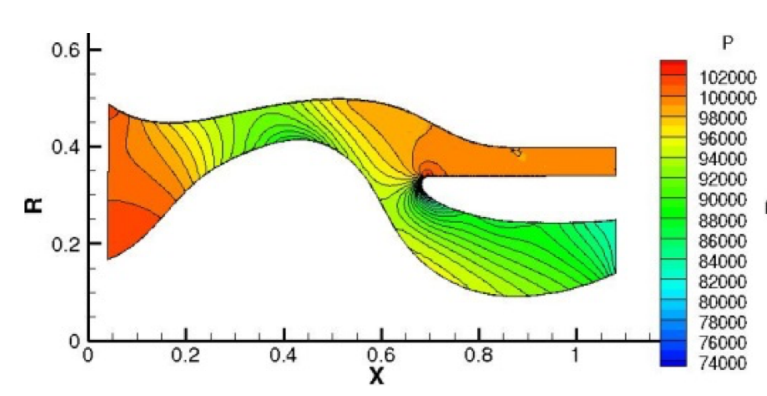

(a) Pressure of gas phase

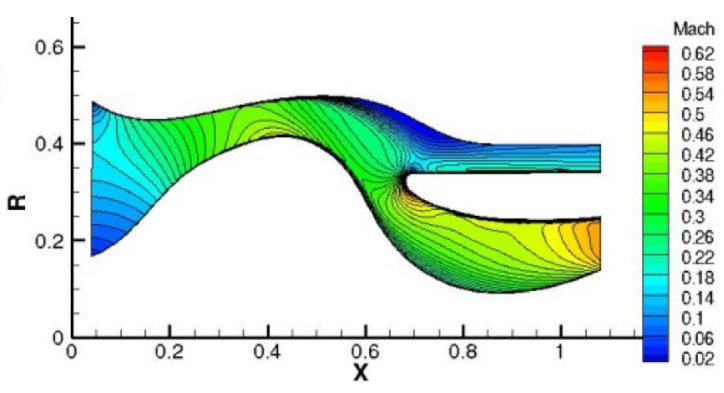

(b) Mach number of gas phase

Figure 6. Contours of pressure and Mach number of gas phase in the IPS model

\section{B. Geometry and gas phase of a generic IPS}

The generic IPS geometry used in the simulation is based upon the axisymmetric advanced IPS configuration that was studied by Hamed et al. ${ }^{16}$ The unstructured computational mesh was generated by processing a structured multi-block body-fitted grid in order to minimize grid related numerical diffusion. The IPS was modelled as a 3D sector domain of the full annulus with symmetry walls. The cross-section of the axisymmetric geometry can be seen in Fig. 6. The flow enters the gas path, which is bounded by the hub (lower radius wall) and casing (higher radius wall), from the left boundary and then split into the engine core flow (lower outlet) and scavenge (upper outlet) on the right. Figure 6 shows the pressure and Mach number contour of the gas phase as computed by CFD simulation using RANS k- $\varepsilon$ model. A subsonic inlet is used in the simulation, and the exit boundaries are set such that $25.8 \%$ of the mass flow passes through the scavenge flow path to match the flow field reported by Hamed et $\mathrm{a}^{16}$. The flowfield was chosen as representative air flow through an IPS that is characterised by recirculating low momentum region on the casing side near the splitter nose that is the inlet to the scavenge geometry. The flow field was chosen prior to any particle simulations. As such, it was not intended to highlight certain behaviour other than a representative of a realistic sand particle flow in an IPS. In this paper the particle trajectory in the IPS are predicted by the new Eulerian method VR-QMOM and, for comparison, with a Lagrangian method. It is worth nothing that the simple axisymmetric nature of this simplified IPS model means a straight forward Lagrangian model can be 
performed, which consume 1 order of magnitude less CPU time than that of VR-QMOM. However, this simplified case neglected the complexity of a real IPS geometry as shown in Fig. 1, in which the scavenge duct is not only axisymmetric but also made up of torturous flow path that require a less straight-forward Lagrangian model in order to ensure enough number of sand particle arrived in the duct in order to get a statistically conclusive result.

\section{Sand distribution in a generic IPS with elastic surfaces}

For the particle phase, it is assumed that the sand particles in the IPS consist of hard silica particles with density of $2444 \mathrm{~kg} / \mathrm{m}^{3}$. The flow rates of the particles in all the following studies are the same. In this section, wall boundary conditions for the particles are set by assuming perfectly elastic collisions.

The particle motions in the IPS are mainly affected by the gas phase carrier and the inertial character of the particle wall bouncing mechanics. The fine particles will follow the flow path of the gas phase carrier. The large particles are separated from the gas flow by the inertia of the particles and the confinement of the surface. Four sizes of particles are simulated: $3 \mu \mathrm{m}, 10 \mu \mathrm{m}, 200 \mu \mathrm{m}$ and $1000 \mu \mathrm{m}$. Particle trajectories and separation efficiency are investigated from the simulations.

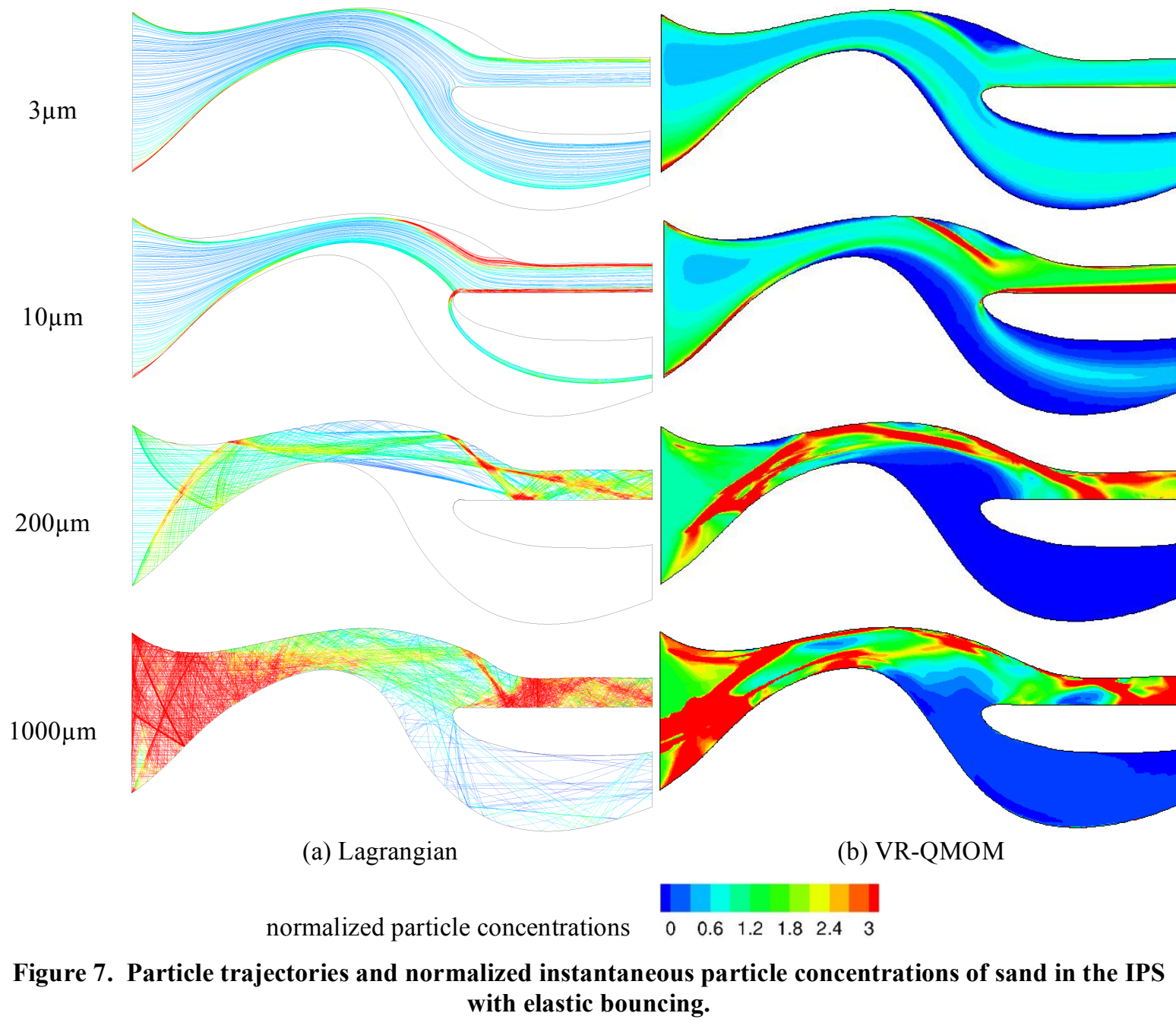

In Fig. 7(a), the particle trajectories are predicted by the Lagragnian method and the particle flow trajectories are coloured by the local particle concentration normalized by the concentration at inlet. With the Lagrangian method, the particle trajectory shows the flow path of each particle injected from the inlet normally to the inlet surface. For $3 \mu \mathrm{m}$ particles, the particle flow is dominated by the gas phase, the particles flow along the flow path of the gas phase. Some of the small particles leave the IPS from the scavenge path. From the particle trajectories of the $200 \mu \mathrm{m}$ particles, the particles bounce off the surface and the trajectories cross in the centre of the flow domain. The particle inertia controls the flow of the particles. Almost all the $200 \mu \mathrm{m}$ particles can be separated. The simulated separation 
efficiency is about $100.0 \%$ of the IPS for $200 \mu \mathrm{m}$ particles by the Lagrangian method. It should be noted that that large particle trajectory, and hence the separation efficiency, is predominantly determined by a combination of wall bouncing characteristics and the wall countouring since these particles behave mostly in a ballistic manner. Since the IPS geometry was not designed to have an elastic wall, the IPS contour actually failed to separate all of the $1000 \mu \mathrm{m}$ particles. The $1000 \mu \mathrm{m}$ particles rebound in the path of IPS and mostly leave the flow path of the gas phase, which makes the flow path of the particles disordered in the IPS. Therefore, the large particles such as with $1000 \mu \mathrm{m}$ diameter may enter the engine through the core path. For the $10 \mu \mathrm{m}$ case, the inertia of the particles is seen to carry most of them directly into the scavenge duct. High concentrations are observed on the lower surface of the separator intake and in the low flow velocity region near the splitter nose, both at the edge of the re-circulation bubble and the upper radius wall of the splitter. Some of the particles impact the nose of the splitter and rebound into the core stream.

In Fig. 7(b), the particle concentrations are predicted by the new VR-QMOM. The particle concentration (found by number densities of both of the two nodes, $n_{1}$ and $n_{2}$ ) is nomalized by the particle concentration at the inlet. It can be seen that all the major features observed in the Lagrangian results have been reproduced with this Eulerian method. Near the inlet, the particle concentrations increase near the hub surface and the outer surface of the separator. This is as a result of particles bouncing off the surface. For larger particles with $200 \mu \mathrm{m}$ diameter, those bouncing off the surface reach the opposite wall of the IPS, which results in an increase of particle concentration in the center of the IPS. This result would not be possible using a basic single dispersed phase velocity Eulerian method. In the throat zone, the particle stream at high number density is observed to collide on the outer surface and reflect back, as seen with the particle trajectories simulated by the Lagrangian method. Two particle streams cross near the recirculating region before the scavenge path. The rebounded particles raise the particle concentration in the scavenge flow path. In the sub-figure showing particle concentration of $10 \mu \mathrm{m}$ particles, features seen in the Lagrangian simulation are again reproduced well, including the stream of particles which enter the core after colliding with the splitter nose. The particle concentration in the core path of $1000 \mu \mathrm{m}$ case is about one tenth of the injecting particle concnetration which at the same order of magnitude of the prediction by Lagrangian method in Fig. (a). The prediction of the particle concentration in the core path shows the possibility of the particles entering the engine core in values directly by the Eulerian method, VR-QMOM.

From the results above we see that the new Eulerian method is able to predict the trajectory crossing and the wall-bouncing of particles. The advantage of the Eulerian simulation is that the particle concentration can be read directly from the simulation as a variable in the transport equations. However, when using the Lagrangian method the particle concentration has to be calculated from the particle trajectory and numbers of particles in each cell using post-processing. These concentrations will depend on the number and injection location of particles used in the simulation. As can be seen in Fig. 7, the particle concentration displayed by a finite number of particles in the Langrangian figures cannot represent the local information continuously. Therefore, a large number of particles have to be injected into the flow domain in order to give sufficient statistical information for the particle distribution in the whole domain ${ }^{16}$.

Table 1. Separation efficiency of particles in generic IPS with elastic surface

$\begin{array}{ccccc}\text { Separation efficiency } & \mathbf{3 \mu m} & \mathbf{1 0} \boldsymbol{\mu m} & \mathbf{2 0 0} \boldsymbol{\mu m} & \mathbf{1 0 0 0} \boldsymbol{\mu m} \\ \text { Lagrangian } & 32.29 \% & 76.06 \% & 100.0 \% & 83.20 \% \\ \text { VR-QMOM } & 34.72 \% & 74.70 \% & 100.0 \% & 94.12 \%\end{array}$

The separation efficiency is calculated by the mass flow rate of the scavenge particle stream to the inlet particle stream.

$$
\eta=\frac{\text { mass flow rate of particles at scavenge path }}{\text { mass flow rate of particles at inlet }}
$$

In table 1, the separation efficiencies are listed from both Lagrangian method and Eulerian VR-QMOM simulations. Again it should be noted that while the exact number found from the Lagrangian method will be affected by the number of particles used, the VR-QMOM method will not. The separation efficiencies for large particles such as $200 \mu \mathrm{m}$ diameter are predicted to be $100 \%$ with both methods. However, if the particle inertia mostly controls the flow path such as of $1000 \mu \mathrm{m}$ particles, the separation efficiency reduces. Fine particles are unlikely to be totally separated by the IPS. The particles of $3 \mu \mathrm{m}$ are only about $30 \%$ separated, which is close to the air mass flow split of $25.8 \%$. About $80 \%$ of $10 \mu \mathrm{m}$ particles leave the engine through the scavenge path with those entering the core having bounced from the splitter nose. The prediction efficiencies of the Lagrangian method and VR-QMOM method are 
quite close to each other. Although the coefficient of restitution based model and the particle momentum equations are equivalent between the Eulerian and Lagrangian method, some differences between the two is to be expected due to the different numerical treatments that must necessarily be used and the different sources of error. For example the Lagrangian result will be affected by the number of particles while the Eulerian method will have errors due to numerical diffusion. However, while taking this into consideration the agreement between the two methods is highly promising.

\section{Sand particle distribution in a generic IPS coated with M246 alloy}

The collision mechanisms and rebound data of the particle surface interaction has been previously investigated by experiments ${ }^{8,10}$. The restitution coefficient of particle-wall interactions is affected by various elements such as the particle properties of density, diameter, shape, material and hardness as well as the geometry and surface characteristics of the IPS. These, together with the flow conditions of the injecting particles such as impingement angles and velocities, affect how the particles behave. The particle-wall interaction mechanism is related to the impingement angle and velocity as well as the physical properties. In this work we neglect the effect of particle size and speed on restitution coefficient but consider the effect of restitution coefficient being a function of impingement angle.

Hamed and Tabakoff $^{13}$ studied the restitution coefficient by measuring particle bouncing in a high temperature facility for different alloys and coatings. Figure 8 shows the rebound data of sand particles from a

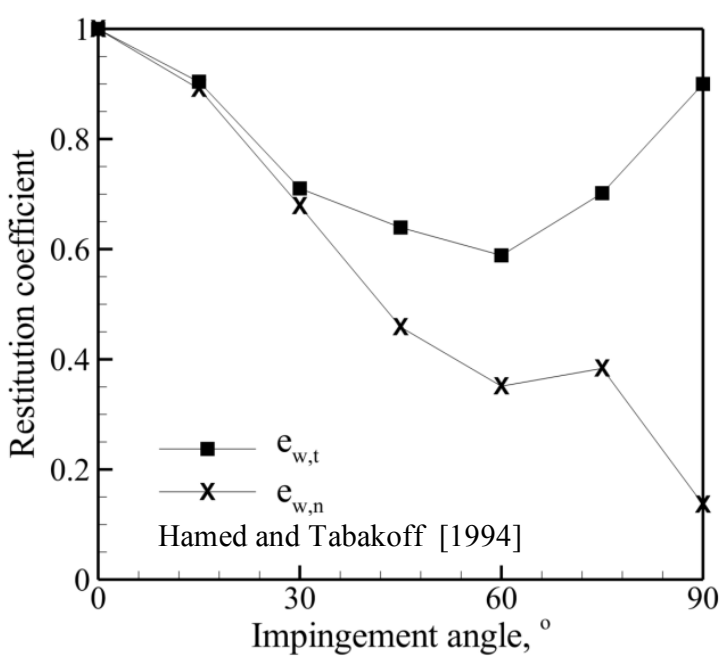

Figure 8. Restitution coefficient variation with impingement angle $\beta_{i m}{ }^{13}$

M246 alloy coating as a function of the impingement angle. The restitution coefficient $\mathrm{e}_{\mathrm{wn}}$ is the ratio of normal velocities of the injecting particles to reflected particles, and $\mathrm{e}_{\mathrm{wt}}$ is the ratio of tangential velocities. Zero degree impingement represents particles sliding along the surface. As such, both restitution coefficients are unity at zero degree impingment.

Figure 9 shows the particle trajectories modeled by the Lagrangian method and the normalized particle concentration of sands calculated by the VR-QMOM. In these simulations the particle-wall coefficients of restitution vary according to the impingement angle using the data in Fig. 8. The restitution coefficients are less than unity and the rebound angles of the particle changes accordingly. The speed of the sand flow is reduced in the collision with the coating surface and the reduction enhances the influence of the gas carrier on the particle flow. As for the elastic case, a very good agreement is seen between Lagrangian and Eulerian simulations for all particle sizes. The VRQMOM method has also successfully reproduced the changes to the particle flow caused by the changed surface properties. As seen in the concentration distribution of $200 \mu \mathrm{m}$ particles, for example, the location of the particle stream generated from the hub surface colliding with the outer surface has moved downstream in the coated IPS compared with distributions in Fig. 7. The $30 \mu \mathrm{m}$ case is particularly interesting as it shows that the particles bounce off the hub surface with a low trajectory before collecting in the low speed re-circulation region located above the splitter where a high concentration is observed.

The surface bouncing mechanisms are different between large particles and small particles. For small particles, whose particle flow path are more closely following the gas phase, the impingement angle will be lower meaning that particles are more likely to be directed towards the flow path of the gas phase. This will reduce the separation efficiency of small particles as seen in $3 \mu \mathrm{m}$ case. For the large particles whose flow path is mainly controlled by the particle inertia, the effect of the inelastic surface provides a better match to the IPS wall contour. For the large particles over $500 \mu \mathrm{m}$ which were hard to remove totally when a fully elastic wall was used, the separation efficiency may be increased. For particles in $1000 \mu \mathrm{m}$ diameter, the separation efficiency was raised to $100 \%$ in the IPS coated with M246.

In Tables 1 and 2, the separation efficiencies of particles are listed for the IPS assumed with elastic surface and the IPS coated with M246 alloys. The separation efficiencies of $3 \mu \mathrm{m}, 10 \mu \mathrm{m}, 200 \mu \mathrm{m}$ and $1000 \mu \mathrm{m}$ are calculated with both simulation methods for fully elastic and M246 alloy bouncing conditions. For the elastic case the flow path of the $3 \mu \mathrm{m}$ particles are close to the gas phase and about $32 \%$ particles leave through the scavenge path, this 
being close to the splitting ratio of the gas phase (25.8\%). Coated with M246 alloy, the separation of $3 \mu \mathrm{m}$ particles has been reduced to around $30 \%$. The largest particles of $1000 \mu \mathrm{m}$ are $100 \%$ separated eventually.

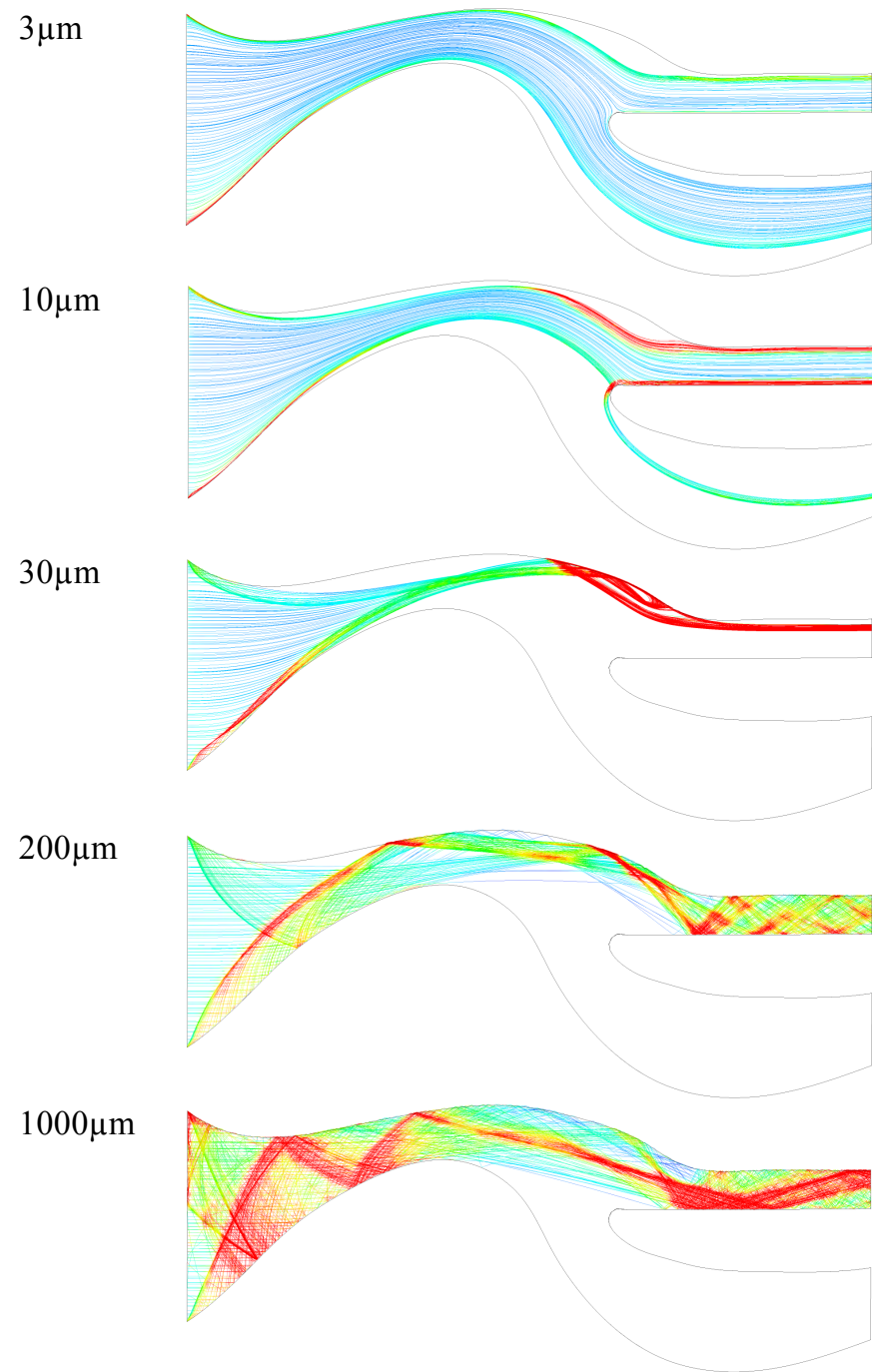

(a) Lagrangian
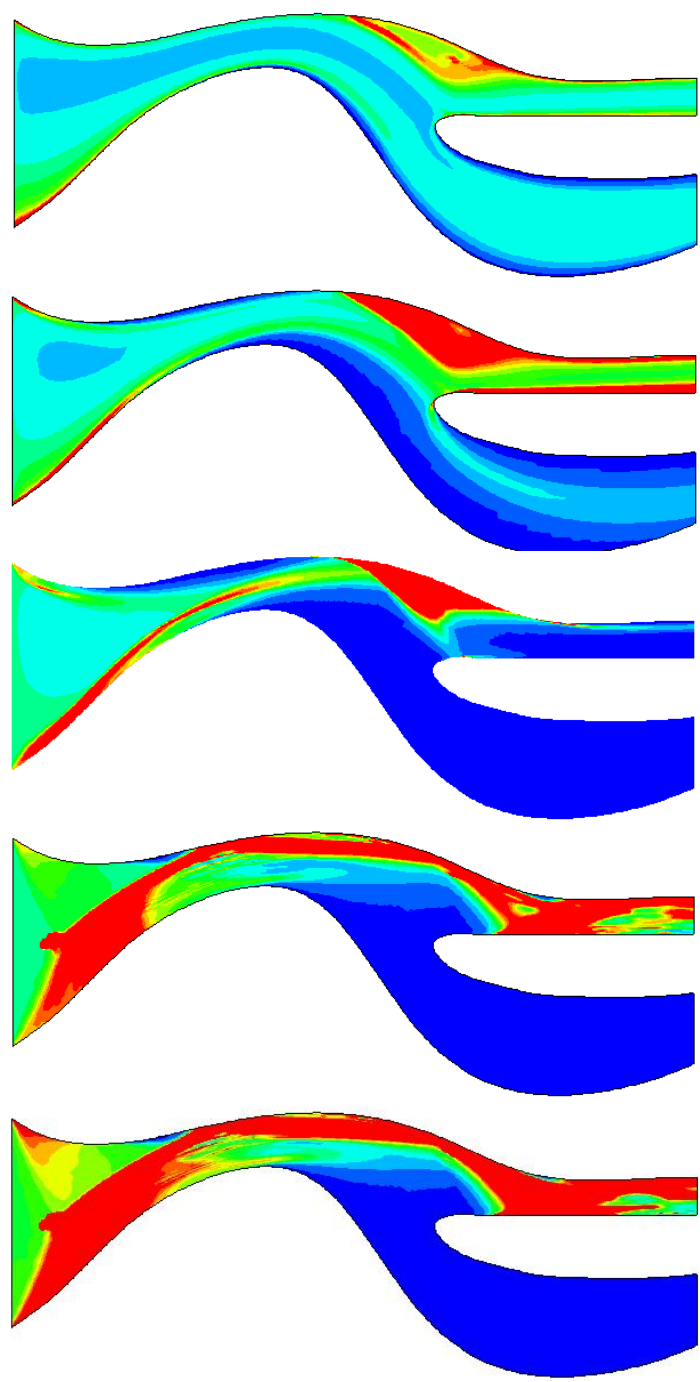

(b) VR-QMOM

normalized particle concentrations

Figure 9. Particle trajectories and normalized instantaneous concentrations of sand in the IPS with M246 alloy coating

Table 2. Separation efficiency of particles in the IPS with M246 alloy coating

$\begin{array}{ccccc}\text { Separation efficiency } & \mathbf{3 \mu m} & \mathbf{1 0} \boldsymbol{\mu m} & \mathbf{2 0 0} \boldsymbol{\mu m} & \mathbf{1 0 0 0} \boldsymbol{\mu m} \\ \text { Lagrangian } & 30.00 \% & 75.67 \% & 100.0 \% & 100.0 \% \\ \text { VR-QMOM } & 31.02 \% & 76.55 \% & 100.0 \% & 100.0 \%\end{array}$

Figure 10 shows a summary all the separation efficiency of particles in the IPS with M246 alloy coating at the whole range of particle size distributions listed in the Fig. 1. The separation efficiency of particles larger than $20 \mu \mathrm{m}$ are $100 \%$. As expected the smaller particles have a lower separation efficiency. The separation efficiency of $1 \mu \mathrm{m}$ particles is near to the splitting ratio of the gas phase. 


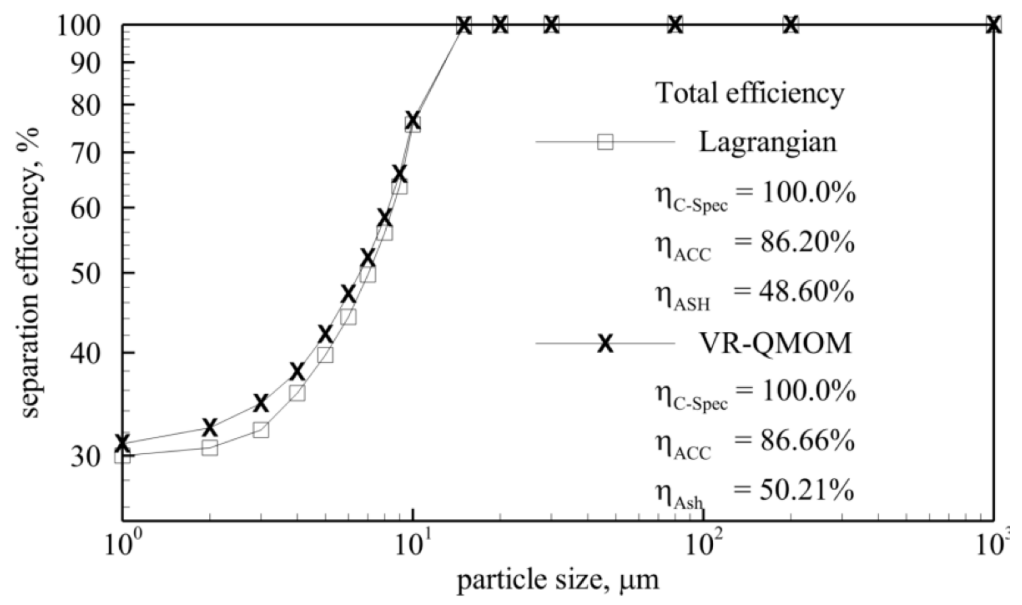

Figure 10. Separation efficiency of particles simulated by Lagrangian method and VR-QMOM

In order to fully assess the output of the VR-QMOM method a comparison with Lagrangian results for realistic particle size distributions was made. Here two standard sand specifications that are commonly used for certification in desert environment operations - namely C-Spec and AC Coarse (ACC) - and a widely used ash representation that is described in Figure 1 are used. The aim is to assess whether the differences that have been observed before for single particle sizes would lead to a different prediction of the overall IPS efficiency. The total efficiency is calculated by the monodisperse separation efficiencies weighted by the particle size distribution. The C-Spec particle spectrum includes particles in the diameter range from 40-800 $\mu \mathrm{m}$. Calculated total efficiencies by both Lagrangian and VR-QMOM methods are equal to $100 \%$. The ash particles consist of fine particles with diameter in the rage 1-25 $\mu \mathrm{m}$, which can represent the most damaging fine vocanic ash encountered at high altitude for commerical aircraft operation. The calculated efficiencies are near to $50 \%$. The total efficiency of separation simulated by the Lagrangian method is $\eta_{\text {ash,Lag }}=48.60 \%$, and $\eta_{\text {ash,VR-QMOM }}=50.21 \%$ calculated by the VR-QMOM. AC Coarse covers a large range of particles from 1-200 $\mu \mathrm{m}$, including both fine particle and coarse particles. The calculated separation efficiency are $86.45 \%$ by Lagrangian method and $86.56 \%$ by VR-QMOM respectly. It is observed that the separation efficiency simulated by the Eularian VR-QMOM is in all cases very close to the Langrangian method, which has previously been shown to give accurate predictions of separation efficiency for IPS of helicopters. Hence the new VR-QMOM method is able to accurately predict particle flows dominated by wallparticle interactions.

\section{Conclusion}

In this research, the multiphase dynamics of an inertial particle separator have been investigated for foreign particle ingestion. The motivation of the research is to develop an Eulerian method for multiphase flow which can predict the interactions between the particles and surfaces. If the foreign particles are hard solids such as sand or dust, the basic Eulerian method fails to describe the particle trajectories and distributions as it cannot predict bouncing. A new Eulerian method VR-QMOM model is used here to predict the dilute particle flow in the IPS. The particle flow stream bouncing and crossing in a generic IPS are successfully modeled. The distributions of particles are seen to match those calculated by Lagrangian simulation. The trend with particle size and with surface contact behavior is correctly reproduced

The particle distribution in an IPS coated with M246 alloy are predicted by both VR-QMOM and Lagrangian method. The separation efficiency predicted by the VR-QMOM is very close to the efficiency found by the Lagrangian method in the whole range of particle spectrum, from fine particles of about $1 \mu \mathrm{m}$ to coarse particles of $800 \mu \mathrm{m}$. This enables separation efficiencies for particle size distributions to be found using the monodisperse efficiencies weighted by the size distribution. The ability of the Eulerian VR-QMOM method to predict the behavior of dilute particle flows in an IPS is thus demonstrated by the calculation separation efficiency which is seen to be very close to those found with established Lagrangian methods. This gives confidence that the VR-QMOM method can be successfully applied in other environments where the availability of Eulerian methods will offer significant advantages over Lagrangian methods. These include turbomachinery within the engine core where multiple rotating and stationary frames of reference make efficient use of Lagrangian methods difficult. 


\section{Acknowledgments}

This work has been funded by the Innovate UK (formerly the United Kingdom Technology Strategy Board, TSB) under SILOETII, in conjunction with Rolls-Royce PLC.

\section{References}

\footnotetext{
${ }^{1}$ Wadcock A. J., Ewing L. A., Solis E., Potsdam M., Rajafopalan G., "Rotorcraft Downwash Flow Field Study to Understand the Aerodynamics of Helicopter Brownout," American Helicopter Society Southwest Region Technical Specialists' Meeting, Dallas-Fort Worth, TX, 2008.

${ }^{2}$ Brun K., Nored M., Kurz R., "Particle Transport Analysis of Sand Ingestion in Gas Turbine Engines," Journal of Engineering for Gas Turbines and Power, Vol. 134, 2012, pp. 012402.

${ }^{3}$ Mund M. G., Guhna H., "Gas Turbine Dust Air Cleaners," Gas Turbine Division of The American Society of Mechanical Engineers for presentation at the ASME Gas Turbine Conference \& Products Show, ASME, 70-GT-104, 1970.

${ }^{4}$ Sirs R. C., "The Operation of Gas Turbine Engines in Hot \& Sandy Conditions-Royal Air Force Experiences in the Gulf War," AGARD Conference Proceedings, AGARD-CP-558-2, 1994.

${ }^{5}$ Dunn M. G., Baran A. J., Miatech J. "Operation of Gas Turbine Engines in Volcanic Ash Cloud," Transactions of the $A S M E$, Vol. 118, Oct. 1996, pp. 724-731.

${ }^{6}$ Lynch F.T., Khodadoust A., "Effects of ice accretions on aircraft aerodynamics," Progress in Aerospace Sciences, Vol. 37, 2001, pp.669-767.

7 Tabakoff, W., "Review of Material Erosion Exposed to Aerodynamic Conditions," U.S. Dept. of Engineering, Rept. on Program Review, Oak Ridge, 1986.

${ }^{8}$ Hamed A., Tabakoff W., "Erosion and Deposition in Turbomachinery", Journal of Propulsion and Power, Vol. 22, No. 2, 2006, pp. 350-360.

${ }^{9}$ Tan S. C., Elder R. L., Hams P. K., "Particle Trajectories in Gas Turbine Engines", AGARD Conference Proceedings, AGARD-CP-558-14, 1994.

${ }_{10}$ Abbedi, M., "Effect of Restitution Coefficient on Inertial Particle Separator's Efficiency", Master Dessertaition, Department of Mechanical and Industrial Engineering, Northeastern University, Boston, 2009.

${ }^{11}$ MIL-SPEC. MIL_E_5007D, 1973.

12 Duffy R. J., "Design and Component Test of Engine Air Inlet Particle Separator for the CV-7A Aircraft", USAAVLABS Techinical Report, 1968, pp. 68-48.

${ }^{13}$ Hamed A., Tabakoff W., "Experimental and Numerical Simulations of the Effects of Ingested Particles in Gas Turbine", Propulsion and energetics panel symposium. Rotterdam, The Netherlands, 1994

${ }^{14}$ Saeed F., Al-Garni A. Z., “Analysis Method for Inertial Particle Separator,” Journal of Aircraft, Vol. 44, No. 4, 2007 , pp. 1150-1158.

${ }^{15}$ Filippone A., Bojdo N., "Turboshaft engine Air Particle Separation,” Progress in Aerospace Sciences, Vol.46, 2010, pp. 224-245

${ }^{16}$ Hamed A., Jun Y. D., Yeuan J. J., "Particle Dynamics Simulations in Inlet Separator with an experimentally Based Bounce Model," Journal of Propulsion and Power, Vol. 11, No. 2, 1995, pp. 230-235.

${ }^{17}$ Barone D., Loth E., "Fluid Dynamics of an Inertial Particle Separator," in 52nd AIAA SciTech, no. 2014-1314. AIAA, 2014.

${ }^{18}$ Barone D., Loth E.,Snuder P.H., "Particle Dynamics of a 2-D Inertial Particle Separator", Proceedings of ASME Turbo Expo 2014: Turbine Technical Conference and Exposition, GT2014-26922, ASME, 2014.

${ }^{19}$ de la Calzada P., Vazquez R., Fernandez F., and San Segundo M. P. G., "Particle Dynamics Simulation for Aeroengine Intake Design,” Industria de Turbo Propulsores, Tech. Rep. ISABE 99-7280, 1998.

${ }^{20}$ Fox R.O., "Higher-order Quadrature-based Moment Methods for Kinetic Equations," Journal of Computational Physics, Vol. 228, 2009, pp.7771-7791.

${ }^{21}$ Vikas V., Wang Z. J., Passalacqua. A., Fox. R. O. "Realizable High-order Finite-volume Scheme for Quadrature-based Moment Methods", Journal of Computational Physics, Vol. 230, 2011, pp. 5328-5352

${ }^{22}$ Desjardins O., Fox R. O., Villedieu P., "A Quadrature-based Moment Method for Dilute Fluid-particle Flows”, Journal of Computational Physics, Vol. 227, 2008, pp. 2514-2539

${ }^{23}$ Sun D., Garmory A., Page. G, "Velocity-reconstructed Two-node Quadrature Method of Moment for Dilute Fluid-particle Flow," Journal of Computational Physics. (to be submitted)

${ }^{24}$ FLUENT. "ANSYS FLUENT 12.0 Theory Guide". ANSYS FLUENT 12.0 Theory Guide, 2012.

${ }^{25}$ Lapworth, B., "Hydra CFD: A Framework for Collaborative CFD Development", International Conference on Scientific and Engineering Computation (IC-SEC), 2004

${ }^{26}$ Guha A. "A Unified Eulerian Theory of Turbulent Deposition to Smooth and Rough Surfaces," Journal of Aerosol Science, Vol.28, No. 8, 1997, pp. 1517-1537

${ }^{27}$ Schiller L., Nauman A., “A Drag Coefficient Correlation”, V.D.I. Zeitung Vol. 77, 1935, pp. 318-320.
} 